\title{
Looking for molecular gas in a massive lyman break galaxy at $\mathrm{z}=\mathbf{4 . 0 5}$
}

\author{
Qinghua $\operatorname{Tan}^{1,2}$, Emanuele Daddi ${ }^{1}$, Mark Sargent ${ }^{1}$, Jackie Hodge ${ }^{3}$, \\ and $\mathrm{Yu} \mathrm{Gao}^{2}$ \\ ${ }^{1}$ CEA, Laboratoire AIM, Irfu/SAp, F-91191 Gif-sur-Yvette, France \\ email: edaddi@cea.fr \\ ${ }^{2}$ Purple Mountain Observatory, CAS, 2 West Beijing Road, 210008 Nanjing, China \\ ${ }^{3}$ Max-Planck Institute for Astronomy, Königstuhl 17, 69117 Heidelberg, Germany
}

Abstract. We present a search for CO emission in a massive lyman break galaxy at $\mathrm{z} \sim 4.05$.

Keywords. galaxies: high-redshift, galaxies: star formation, radio lines: galaxies

We have observed the $\mathrm{CO}(4-3)$ and $\mathrm{CO}(6-5)$ lines with the Plateau de Bure Interferometer. The observations of each individual configuration show a tentative detection at the $\sim 3 \sigma$ level of $\mathrm{CO}$ emission at the position of the ACS/HST source. The signal is improved to $\mathrm{S} / \mathrm{N} \sim 5$ when combining $\mathrm{CO}(4-3)$ and $\mathrm{CO}$ (6-5) observations (Fig. 1). We have run extensive simulations to estimate that the chance probability of such a signal in our combined datacubes is $\sim 2 \times 10^{-4}$. Assuming that both detections are real, we infer a molecular gas mass of $\sim 1.4 \times 10^{11} \mathrm{M} \odot$ by adopting a conversion factor of $\alpha_{\mathrm{CO}} \sim$ 7.0, which is based on the $\alpha_{\mathrm{CO}}$ - metallicity relation (Magdis et al. 2011; Sargent et al. $2012 \mathrm{~b})$. The location of this galaxy in the $L_{\mathrm{IR}}-L_{\mathrm{CO}}^{\prime}$ plane suggests little variation from the trend defined by normal star-forming galaxies over $0<z<2.2$, possible evidence against a too strong evolution of the conversion factor to higher redshifts. The molecular gas ratio $(\sim 68 \%)$ is found to be comparable to the ratios observed at $z=2$ (Magdis et al. 2012a), providing additional support for the existence of a plateau in the redshift evolution of the specific SFR of normal galaxies at $z>3$. However, we need more CO observations to make a definitive detection and thus further confirm these conclusions.
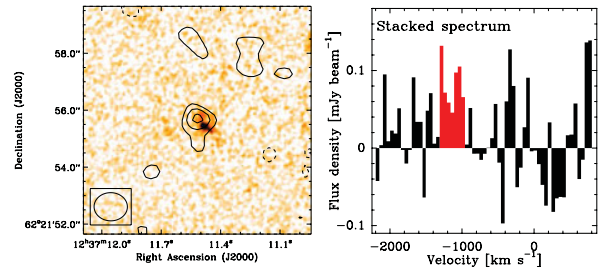

Figure 1. Left: Countours of stacked CO (4-3) and CO (6-5) overlaid on HST+WFC3 F140W image. Countour levels start at $\pm 2 \sigma$ and are in steps of $1 \sigma$, with positive(negative) countours shown as solid (dashed) lines. Right: Combined CO spectrum adopting average line ratio from GN20 and M82 total SLED models. The red color indicates the maximum emission region.

\section{References}

Magdis, G., Daddi, E., Elbaz, D., et al. 2011, ApJ, 740, 15

Magdis, G., Daddi, E., Sargent, M., et al. 2012a, ApJ, 758, 9

Sargent, M., et al. 2012b, in preparation 